\title{
Fraktionierung und Klassifizierung menschlicher Immunglobuline und ihre Reaktion mit sessilen Antigenen beim bullösen Pemphigoid
}

\author{
Von H. Bockendahe, W. Remy und Teda Peters
}

Aus der Hautklinik (Direktor: Prof. Dr. med. G. Stüttgen) der FreienUniversität Berlin im Rudolf-Vircbonv-Krankenbaus

(Eingegangen am 13. August 1971/29. April 1972)

1. Aus den Seren von fünf an bullösem Pemphigoid erkrankten Patienten und einem Pemphiguskranken wurde mittels Fällungsreaktionen die $\gamma$-Globulin-Fraktion gewonnen, die ihrerseits über eine DEAE-Sephadex-Säule fraktioniert wurde.

2. Durch Anwendung der indirekten Immunfluoreszenz konnte die Antigen-Antikörper-Reaktion an der Basalmembran bestimmten Ig G-Fraktionen zugeordnet werden.

3. Die Berechnung einer „,spezifischen Fluoreszenz" (Quotient aus dem reziproken Verdünnungstiter und der Ig G-Konzentration der Untersuchungslösung) ergab, daß nur cin Bruchteil des im Serum vorhandenen Ig G mit dem fixierten Antigen reagiert.

4. Das aktive Eiweiß konnte auf das 82-fache des Ausgangswertes im Serum angereichert werden.

5. Die Patienten mit bullösem Pemphigoid zeigten eine deutliche Erhöhung des Mittelwertes von Ig A und Ig G im Serum. The fractionation and classification of buman immunoglobulins and their reaction with sessile antigens present in cases of bullous
pemphigoid

1. The $\gamma$-fraction was obtained from the sera of five patients with bullous pemphigoid and one patient with pemphigus by fractional precipitation and further fractionated on a DEAE-Sephadex column.

2. The antigen-antibody reaction was located on the basal membrane of certain Ig G-fractions by indirect immunofluorescence.

3. The calculated "specific fluorescence" (ratio of the reciprocal dilution titre and the Ig G-concentration of the test solution) shows that only a fraction of the Ig G present in the serum reacts with the fixed antigen.

4. The active protein was enriched 82 -fold with respect to the starting value of the serum.

5. The patients with bullous pemphigoid showed markedly elevated average serum values for $\operatorname{Ig} A$ and $\operatorname{Ig} G$.

Beutner und Mitarbeiter (1) beschrieben 1964 zum ersten Male, daß Antikörper von Patienten mit Pemphigus vulgaris im Stratum spinosum bestimmter epithelialer Gewebe fixiert werden. 1965 zeigten BEUTNER und Mitarbeiter (2), ebenfalls mittels der Methode der indirekten Immunfluoreszenz, das Vorkommen von gegen die Basalmembran der Epidermis gerichteten Antikörpern bei klinisch bzw. histologisch als bullöses Pemphigoid diagnostizierten Fällen. Seit dieser Zeit sind die Beobachtungen vielfach bestätigt worden, und eine Diskussion über die Frage, ob der Pemphigus bzw. das bullöse Pemphigoid eine Autoimmunerkrankung sei, schloß sich daran an. Eine zusammenfassende Übersicht über das PemphigoidProblem, wie es sich heute darstellt, findet sich bei SAMs (3), der folgende Punkte herausstellt:

1. Der Patient besitzt einen gegen die Basalmembran gerichteten Antikörper.

2. Dieser Antikörper kann Komplement binden.

3. Sowohl Antikörper als auch Komplement sind am Orte der pathologischen Hauterscheinungen nachweisbar.

4. Polymorphkernige Leukocyten bewirken ein entzündliches Infiltrat.

5. Die Krankheit hat einen chronischen Verlauf.

Die bisherigen Untersuchungen sprechen dafür, daß die Antikörper zur Gruppe der Immunglobuline $G$ gehören. Das Ziel unserer Arbeit war, zunächst eine
Fraktionierung des Ig G durchzuführen, wobei eine Isolierung der für die Antigen-Antikörperreaktion verantwortlichen Fraktion beabsichtigt war.

Für die Durchführung dieser Experimente standen uns sieben Patienten zur Verfügung, von denen fünf an histologisch gesichertem bullösen Pemphigoid litten und der sechste die klinischen Symptome eines Pemphigus foliaceus aufwies. Im Serum eines weiteren Kranken mit klinisch und histologisch gesichertem bullösen Pemphigoid waren mit der direkten und der indirekten Immunfluoreszenzmethode keine Basalmembran-Antikörper nachweisbar.

Derartige Beobachtungen sind in der Literatur öfter beschrieben worden. Dabei erhebt sich die Frage, ob, wie gelegentlich erörtert, die Antigen-AntikörperReaktion erst in einem späteren Stadium der Erkrankung nachweisbar wird, oder ob andere, zur Zeit noch unbekannte Immunglobuline als Antikörper reagieren.

Alle Kranken waren unbehandelt, außer einem $\mathrm{Pa}$ tienten (Patient 1), den wir im Anschluß an einen akuten Schub noch zweimal im therapiebedingten Intervall untersuchen konnten.

\section{Methoden}

Die Isolierung der $\gamma$-Globuline aus dem Serum und deren Chromatographie an DEAE-Sephadex crfolgte nach der von KrCKröFEN und Mitarbeitern (4) beschriebenen Methodik. 
Das Prinzip dieser Methode ist folgendes:

1. Nach Einstellung des $\mathrm{pH}$ des Serums auf 8,0 und hinreichender Verdünnung Zusatz einer wäßr. Rivanollösung.

2. Nach $30 \mathrm{Min}$. Dekantieren des Überstandes und Zugabe von $\mathrm{NaCl}(50 \mathrm{~g} / \mathrm{l})$.

3. Nach 12 Stdn. Entfernen des gefälliten Rivanols durch Zentrifugieren.

4. Nach nochmaliger Verdünnung der Lösung und Justierung des $\mathrm{pH}-$ Wertes auf 7,0 Zugabe des gleichen Volumens 4M $\left(\mathrm{NH}_{4}\right)_{2} \mathrm{SO}_{4}$-Lösung.

5. Nach 16 Stdn. Gervinnung des Niederschlages durch Zentrifugieren, anschließend Waschen mit $2 \mathrm{M}\left(\mathrm{NH}_{4}\right)_{2} \mathrm{SO}_{4}$ und Lösen in phosphatgepufferter physiol. $\mathrm{NaCl}-\mathrm{Lösung}$.

6. Dialysieren gegen. Säulenpuffer $(0,02 \mathrm{M}$ Tris-Phosphatpuffer, $\mathrm{pH} 8,6)$.

7. Isotonische Einengung der Eiveißlösung mit Carbowax nach KOHN (5).

8. Verbringen der Eiweißlösung auf eine DEAE-SephadexSäule.

Wir verwendeten jeweils 30 bis $40 \mathrm{ml}$ Serum pro Versuch. Die Säulengröße betrug $20 \times 2,6 \mathrm{~cm}$.

Die Pufferkonzentrationen im Eluat wurden mit einer PhilipsLeitfähigkeits-Meßbrücke ermittelt.

Den Eiweißgehalt bestimmten wir nach Weichselbaum (6) oder mit dem von Warburg und Christian (7) angegebenen Verfahren.
Die immunfluoreszenzmikroskopischen Untersuchungen erfolgten mit der indirekten Methode in Anlehnung an WeLLER und CooNs (8) und an Beurner $(1,2,9)$ wie folgt:

1. Anfertigung von Gefrierschnitten (Kryostat SLEE) aus frisch excidierter Meerschweinchenzunge oder -lippe von 5 bis $6 \mu \mathrm{m}$ Dicke.

2. Inkubation det Schnitte mit Patientenserum bzw. $\boldsymbol{y}$-GlobulinFraktionen (Verdünnungsreihen).

3. Nach viermaligem Wäschen der Schnitte mit Cooss-Puffer Überschichten mit fluorescein-markiertem Antiserum (Verdünnung $1: 30$ ).

4. Waschen der Schnitte mit Coons-Puffer.

5. Eindecken der Schnitte mit Glycerin-Puffergemisch.

6. Beurteilung der Präparate mit einem Fluoreszenzmikroskop der Firma Zeiss.

7. Kontrollversuche in üblicher Weise.

Immunelektrophöresen zur qualitativen Differenzierung von Plasmaproteinen wurden in Anlehnung an Grabar und Mitarbeiter (10) durchgeführt.

Zur quantitativen Bestimmung der Immunglobuline verwendeten wir Partigen-Platten in Anlehnung an die von Mancrns und Mitarbeitern (11) entwickelte Technik.

Die Partigen-Platten, die exforderlichen Anti-Seren und die mit Fluoresceinisothiocyanat markierten Anti-Humanseren bezogen wir von den Behringwerken, Marburg. Das Di-aethyl-amino-aethyl(DEAE-) Sephadex lieferte die Deutsche Pharmacia, Frankfurt. Das

Tab. 1

Übersicht über alle an Seren von 6 Patienten durchgeführten Versuche. Patient 1 wurde dreimal untersucht (1-1b). Die Eiweißbestimmungen erfolgten unter Verwendung von Partigenplatten und Immunelektrophoresen.

\begin{tabular}{|c|c|c|c|c|c|}
\hline Pat. Nr. & Diagnose & Klin. Status & Fraktion & Fluoreszenz & Eiweiß̣körper \\
\hline 1 & Alterspemphigoid & akutes Stadium & $\begin{array}{l}\text { Serum } \\
\gamma \\
\gamma_{1} \\
\gamma_{2} \\
\gamma_{3}^{\prime}\end{array}$ & $\begin{array}{c}1: 1-1: 40 \\
1: 1-1: 4 \\
1: 1-1: 4 \\
\text { nicht gesammelt } \\
\varnothing\end{array}$ & $\begin{array}{l}\text { Ig A, Ig G, Ig M, } \beta_{1} \text { A } \\
\text { Ig G, Coeruloplasmin } \\
\text { Ig } G, \text { Albumin }^{+} \text {) } \\
\text { Ig } G\end{array}$ \\
\hline $1 \mathrm{a}$ & Alterspemphigoid & $\begin{array}{c}\text { klinisch erscheinungsfrei unter } \\
10 \text { mg Methotrexat/Woche } \\
+ \\
\text { 0,1 DADPS*) pro Tag }\end{array}$ & $\begin{array}{l}\text { Serum } \\
\gamma \\
\gamma_{1} \\
\gamma_{2} \\
\gamma^{\prime}, \\
\gamma^{\prime}, \\
\end{array}$ & $\begin{array}{l}1: 1-1: 40 \\
1: 1-1: 40 \\
1: 1 \text { fraglich } \\
\varnothing \\
1: 1-1: 40 \\
\varnothing\end{array}$ & $\begin{array}{l}\text { Ig A, Ig G, Ig M } \\
\text { Ig A, Ig G, Albumin }{ }^{+} \text {), Coeruloplasmin } \\
\text { Ig G } \\
\text { Ig G } \\
\text { Ig A, Ig G, Albumin } \\
\text { Ig A, Ig G, Albumin u. a. }{ }^{+} \text {) }\end{array}$ \\
\hline $1 \mathrm{~b}$ & Altersemphigoid & $\begin{array}{l}\text { klinisch erscheinungsfrei sonst } \\
\text { s. } 0 .\end{array}$ & $\begin{array}{l}\text { Serum } \\
\gamma \\
\gamma_{1} \\
\gamma_{2} \\
\gamma_{3}^{\prime} \\
\gamma_{4}^{\prime}\end{array}$ & $\begin{array}{l}1: 1-1: 10 \\
1: 1-1: 10 \\
\text { nicht gesammelt } \\
\text { nicht gesammelt } \\
1: 1-1: 10 \\
\varnothing\end{array}$ & $\begin{array}{l}\operatorname{Ig} A, \operatorname{Ig~G}, \operatorname{Ig} M, \beta_{1} A \\
\operatorname{Ig~A,~Ig~G~} \\
\operatorname{Ig~G} \\
\operatorname{Ig~A,~Ig~G~}\end{array}$ \\
\hline 2 & $\begin{array}{l}\text { Alterspemphigoid } \\
\text {. }\end{array}$ & akutes Stadium & $\begin{array}{l}\text { Serum } \\
\gamma \\
\gamma_{1} \\
\gamma_{2} \\
\gamma_{3}^{\prime} \\
\gamma^{\prime} \\
\end{array}$ & $\begin{array}{l}1: 1-1: 20 \\
1: 1-1: 40 \\
1: 1 \\
1: 1 \text { fraglich } \\
\varnothing \\
\varnothing \\
\end{array}$ & $\begin{array}{l}\operatorname{Ig~A,} \operatorname{Ig~G,} \operatorname{Ig} M \\
\operatorname{Ig~A,~Ig~G~} \\
\operatorname{Ig~G} \\
\operatorname{Ig~G} \\
\operatorname{Ig~G} \\
\operatorname{Ig~G}\end{array}$ \\
\hline 3 & Altersphemphigoid & akutes Stadium & $\begin{array}{l}\text { Serum } \\
\gamma \\
\gamma_{1} \\
\gamma_{2}^{\prime} \\
\end{array}$ & $\begin{array}{c}1: 1-1: 32 \\
1: 1-1: 8 \\
\text { nicht gesammelt } \\
\varnothing\end{array}$ & $\begin{array}{l}\text { Ig A, Ig G, Ig M, } \beta_{1} A \\
\text { Ig A, Ig G, Albumin }{ }^{+} \text {), Coeruloplaminn }{ }^{+} \text {) } \\
\text { Ig G }\end{array}$ \\
\hline 4 & Alterspemphigoid & akutes Stadium & $\begin{array}{l}\text { Serum } \\
\gamma \\
\gamma_{2} \\
\gamma_{2}^{\prime} \\
\end{array}$ & $\begin{array}{c}1: 1-1: 10 \\
1: 1-1: 5 \\
\text { nicht gesammelt } \\
1: 1-1: 10\end{array}$ & $\begin{array}{l}\left.\text { Ig A, Ig G, Ig } M, \beta_{1} A, x \text {-Ketten }{ }^{+}\right) \\
\text {Ig A, Ig G } \\
\text { Ig } G, x \text {-Ketten }{ }^{+} \text {) }\end{array}$ \\
\hline 5 & $\begin{array}{c}\text { Alterspemphigoid } \\
.\end{array}$ & akutes Stadium & $\begin{array}{l}\text { Serum } \\
\gamma \\
\gamma_{1} \\
\gamma_{2}^{\prime} \\
\end{array}$ & $\begin{array}{l}1: 1-1: 40 \\
1: 1-1: 40 \\
1: 1-1: 10 \\
1: 1 \text { fraglich }\end{array}$ & $\begin{array}{l}\text { Ig A, Ig G, Ig } M, \beta_{1} A \\
\text { Ig A, Ig G, Coeruloplasmin }{ }^{+} \text {) } \\
\text { Ig G } \\
\text { Ig G }\end{array}$ \\
\hline 6 & Pemphigus foliaceus & akutes Stadium & $\begin{array}{l}\text { Serum } \\
\gamma\end{array}$ & $\begin{array}{l}1: 1-1: 4 \\
1: 1-1: 4\end{array}$ & $\begin{array}{l}\text { Ig A, Ig G, Ig } M, \beta_{1} A \\
\text { Ig A, Ig } G \text {, Coeruloplasmin }{ }^{+} \text {) }\end{array}$ \\
\hline
\end{tabular}


Carbowax (Polyaethylenglykol 20000) erhielten wir von der Fluka Fcinchemikalien GmbH, Eschborn/Taunus. Alle übrigen Chemikalien waren handelsübliche Präparate.

\section{Ergebnisse und Diskussion}

Eine Übersicht über das Patientengut und die von uns durchgeführten Untersuchungen vermittelt die $\mathrm{Ta}$ belle 1. Zur im folgenden verwendeten Nomenklatur sei vorausgeschickt, daß alle Fraktionen, welche die Säule bei geringer Ionenstärke des Puffers durchliefen, als $\gamma_{\mathrm{n}}$ und jene, die erst durch höhere Ionenstärken eluiert werden konnten, mit $\gamma_{n}^{\prime}$ bezeichnet wurden.

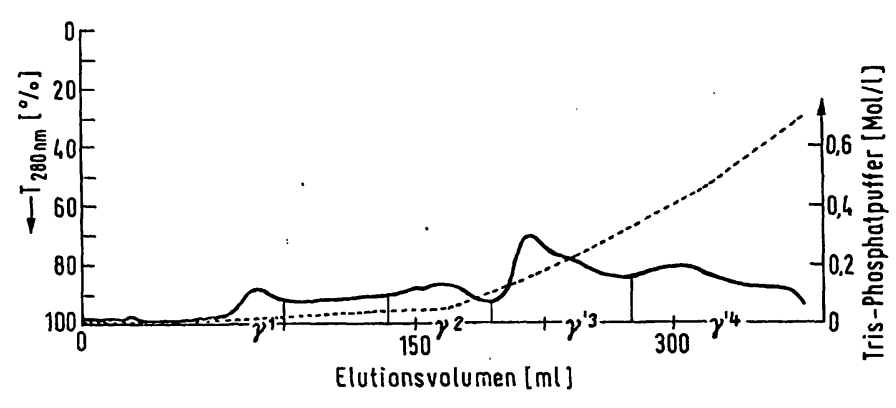

Abb. 1

Elutionsdiagramm $(\rightarrow$ einer DEAE-Sephadex-Säule. Elutionsgradient mit Tris-Phosphatpuffer $(\cdots)$

Abbildung 1 zeigt ein typisches Beispiel für das Elutionsdiagramm einer mit der $\gamma$-Globulinfraktion aus dem Serum des Patienten 2 beschickten DEAE-Sephadex-Säule. Man erkennt mindestens vier verschiedene Fraktionen, die durch bestimmte Ionenstärken des Säulenpuffers charakterisiert sind und in jedem Versuch hinsichtlich der gemessenen Pufferkonzentration reproduziert werden konnten. In einigen Experimenten konnte nur eine $\gamma_{\mathrm{n}}$-Fraktion nachgewiesen werden, oder es fehlte die zweite $\gamma_{n}{ }_{n}$-Fraktion. Die im Eluat bestimmten Pufferkonzentrationen hatten folgende Werte (Mol/1):

$$
\begin{aligned}
& \gamma_{1}: 0,03( \pm 0,025) ; \gamma_{2}: 0,072( \pm 0,002) ; \\
& \left.\gamma_{3}^{\prime}: 0,195 \text { ( } \pm 0,001\right) ; \gamma_{4}^{\prime}: 0,34( \pm 0,042) .
\end{aligned}
$$

In allen vier Fraktionen waren außer dem Ig $G$ andere Eiweiße immunelektrophoretisch meistens nur in Spuren nachweisbar, wobei die $\gamma^{\prime}{ }_{n}$-Fraktionen manchmal größere Beimengungen von Ig A enthielten.

Das Ergebnis einer quantitativen Auswertung eines einzelnen Versuches (Patient $1 \mathrm{~b}$ ) ist in der Tabelle 2 zusammengestellt.

Die Ausbeute der aus den Seren isolierten Eiweißkörper ist nicht groß, entspricht aber der in der Proteinchemie bekannten Größenordnung. Auffallend ist lediglich die Tatsache, daß die Quotienten aus der Summe'der Immunglobuline und dem Gesamteiweiß in den Fraktionen des Säuleneluates kleiner als 1 sind. In vier Versuchen konnten wir für diesen Quotienten in der $\gamma$-Fraktion jedoch einen Mittelwert von 1,05 mit einer kleinen Streuung von $\pm 0,090$ berechnen, so $\mathrm{da} \beta$ die Möglichkeit besteht, da $\beta$ ein Teil der Immunglobuline im Laufe der Säulenchromatographie Ver-
Tab. 2

Tabellarische Übersicht über die Proteinmengen (in $\mathrm{mg}$ ) in den während der Aufarbeitung eines Patientenscrums gewonnenen Fraktionen.

\begin{tabular}{lcccccc}
\hline & $\begin{array}{c}\text { Gesamt- } \\
\text { Eiweiß }\end{array}$ & Ig A & Ig G & Ig $M$ & $\Sigma$ Ig & $\begin{array}{c}\Sigma I g / \\
\text { Gesamt- } \\
\text { Eiweiß }\end{array}$ \\
\hline Serum & 2350 & 104 & 783 & 28 & 915 & 0,39 \\
$\gamma$-Fraktion & 414 & 31,0 & 380 & 0 & 411 & 0,99 \\
$\gamma_{1}$-Fraktion & 5,3 & 0 & 1,8 & 0 & 1,8 & 0,34 \\
$\gamma_{2}$-Fraktion & 34,0 & 0 & 20,3 & 0 & 20,3 & 0,60 \\
$\gamma^{\prime}{ }_{\text {, }}$-Fraktion & 34,2 & 10,3 & 14,0 & 0 & 24,3 & 0,71 \\
$\gamma^{\prime}$-Fraktion & 18,2 & 3,8 & 8,3 & 0 & 12,1 & 0,67 \\
\hline
\end{tabular}

änderungen erfährt und infolgedessen immunchemisch nicht mehr erkannt wird. In den übrigen, nicht zur Ermittlung des Mittelwertes von 1,05 herangezogenen Versuchen beobachteten wir Einzelwerte von 0,39 bis 0,77. Ob die Erklärung in diesem Falle darin liegt, daß entsprechende Abbauprozesse auf einer früheren Stufe des Präparationsganges ablaufen, vermag nicht entschieden $z u$ werden. Merkwürdig ist ein unterschiedliches Verhalten beider Gruppen bei der Immunfluoreszenz, worauf noch eingegangen wird.

Bei der immunfluoreszenzmikroskopischen Untersuchung der einzelnen Fraktionen beobachteten wir in vier Experimenten eine spezifische Basalmembranfluoreszenz - wie sie in Abbildung 2 dargestellt ist -

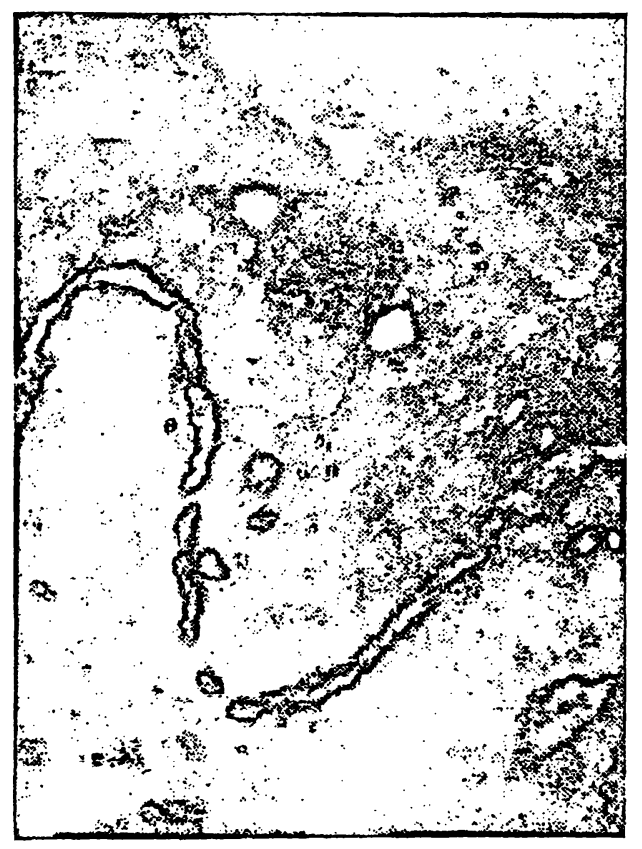

Abb. 2

Spezifische Fluoreszenz der Basalmembran, dargestellt mit der Methode der indirekten Immunfluoreszenz. Substrat: menschliche Epidermis; Patientenserum $\mathrm{Nr}$. 1, unverdünnt; Antiserum: flu
markiertes Ig G, $1: 30$ verdiunnt; Objektiv: 40-fach

mit Hilfe beider $\gamma_{\mathrm{n}}$-Fraktionen und ebensooft unter Verwendung der ersten $\gamma^{\prime}$-Fraktion. Bei zwei Seren ergaben sich insofern Überschneidungen, als positive Ergebnisse sowohl mit der $\gamma_{\mathrm{n}^{-}}$als auch mit der ersten $\gamma^{\prime}{ }_{n}$-Fraktion zur Darstellung kamen, wenn das Resultat auch in beiden Fällen nur in einer Fraktion eindeutig war. Alle Seren, bei denen der Immunglobulin/ 
Eiweißquotient gegen 1 ging, wiesen eine fluoreszenzvermittelnde $\gamma_{n}^{\prime}$-Fraktion auf. In der $\gamma_{4}^{\prime}$-Fraktion fanden sich entsprechende Antikörper trotz Ig G-Nachweis nie.

$\mathrm{Da}$ es sich bei der beobachteten Reaktion nicht um einen unspezifischen Effekt handelt, den jedes Immunglobulin G-Molekül ergibt, läßt sich aus der Tatsache herleiten, daß die Intensität der Fluoreszenz keinesfalls mit der Ig G-Konzentration korreliert werden kann. Wir fanden Fraktionen, in denen trotz relativ hohen Ig G-Gehaltes keine Basalmembran-Antikörper nachweisbar waren, während andere, an der unteren Grenze des Milligrammbereiches, positiv reagierten. Titriert man die Intensität der Fluoreszenz insofern aus, als in Verdünnungsreihen der Ig G-Gehalt soweit herabgesetzt wird, bis keine Fluoreszenz mehr beobachtet werden kann, so ergibt sich ein quantitativer Wert, der den Begriff der „spezifischen Fluoreszenz" ermöglicht. Dieser ist durch den Quotienten aus dem reziproken Titer der Lösung, die gerade noch eine Fluoreszenz bewirkt, und der Ig G-Konzentration in $\mathrm{mg} / 100 \mathrm{ml}$ definiert.

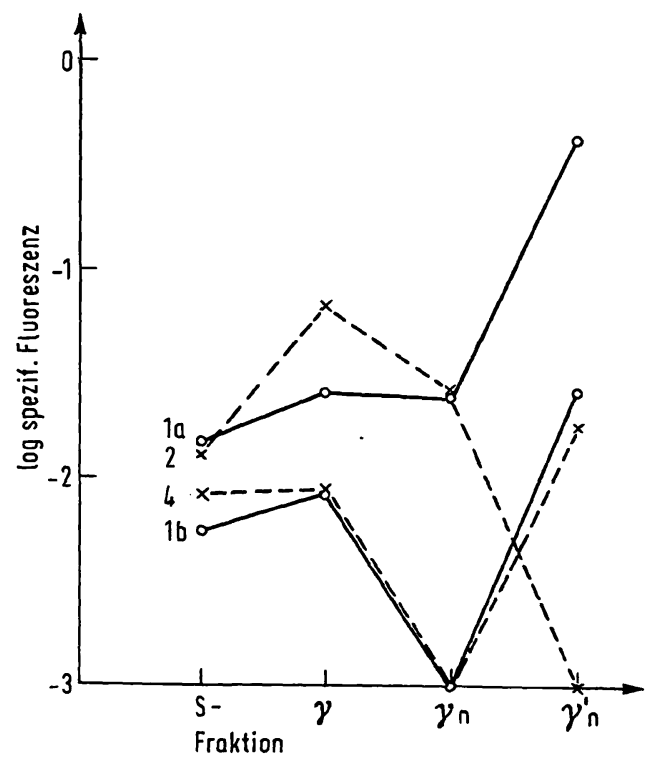

Abb. 3

Logarithmische Auftragung der ,speziftschen Basalmembran- Fluoreszenz" (Ordinate) in den einzelnen Eiweißfraktionen (Abszisse) bei mehreren Präparationsvorgängen. Den Nullpunkt setzten wir willkürlich gleich $\log -3$. Die Zahlenangaben an den Kurven weisen auf die untersuchten Patienten hin (s. Tab. 1).

In der Abbildung 3 findet sich eine derartige Auswertung einiger Versuchsergebnisse. Aus Gründen der Übersichtlichkeit wurde eine logarithmische Darstellung gewählt, wobei auf der Ordinate $\log -3$ willkürlich als Nullpunkt gesetzt wurde. In allen Experimenten ergab sich eine Zunahme der spezifischen Fluoreszenz gegenüber dem im Serum bestimmten Ausgangswert. In einem Versuch erzielten wir eine Anreicherung des aktiven Eiweißes um den Faktor 27, bezogen auf die Ig G-Konzentration im Serum. Bezieht man die Anreicherung auf den Gesamteiweißgehalt des Serums, erhält man einen Faktor von 82.

Untersuchungen des Immunglobulin-Serumspiegels ergaben bei Patienten mit einem bullösen Pemphigoid eine
Tab. 3

Zusammenstellung der Immunglobulin-Konzentrationen (in $\mathrm{mg}$ / $100 \mathrm{ml}$ ) im Serum von an bullösem Pemphigoid erkrankten Personen.

\begin{tabular}{|c|c|c|c|}
\hline Pat. Nr. & $\operatorname{Ig~A}$ & Ig G & $\operatorname{Ig} M$ \\
\hline 1 & 320 & 1500 & 130 \\
\hline $1 \mathrm{a}$ & 376 & 2700 & 96 \\
\hline Ib & 228 & 1820 & 88 \\
\hline 2 & $53 ุ 2$ & 1580 & 120 \\
\hline 3 & 408 & 1360 & 100 \\
\hline 4 & 440 & 1240 & 118 \\
\hline 5 & 172 & 2440 & 190 \\
\hline$\overline{\mathbf{x}}$ & 372 & 1730 & 127 \\
\hline \pm 5 & \pm 123 & \pm 445 & \pm 33 \\
\hline
\end{tabular}

deutliche Erhöhung der Mittelwerte von Ig G und Ig Agegenüber der Norm bei normalen Ig M-Konzentrationen. Eine entsprechende Übersicht vermittelt Tabelle 3.

Der einzige von uns in diesem Zusammenhang beobachtete Pemphiguskranke (Patient 6) zeigte eine Verteilung der Immunglobuline, wie sie von GroB (12) beschrieben wurde. Wir bezogen unsere Werte dabei auf die von Schwartz (13) und FaHEY (14) publizierten Mittelwerte, die mit eigenen Ergebnissen an gesunden Individuen übereinstimmen. Das Fluoreszenzmuster, wie es bei Patienten mit Pemphigus vul-

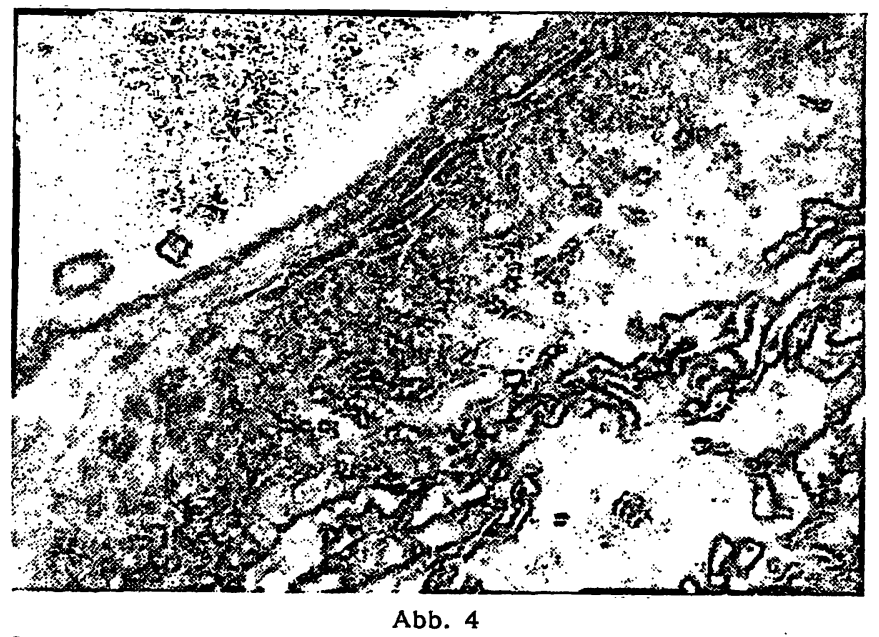

Spezifische Fluoreszenz im Bereich der Zellgrenzen des stratum spinosum der Epidermis, dargestellt mit der indirekten Immunfluoresbildung 2 . Objektiv 10 fach.

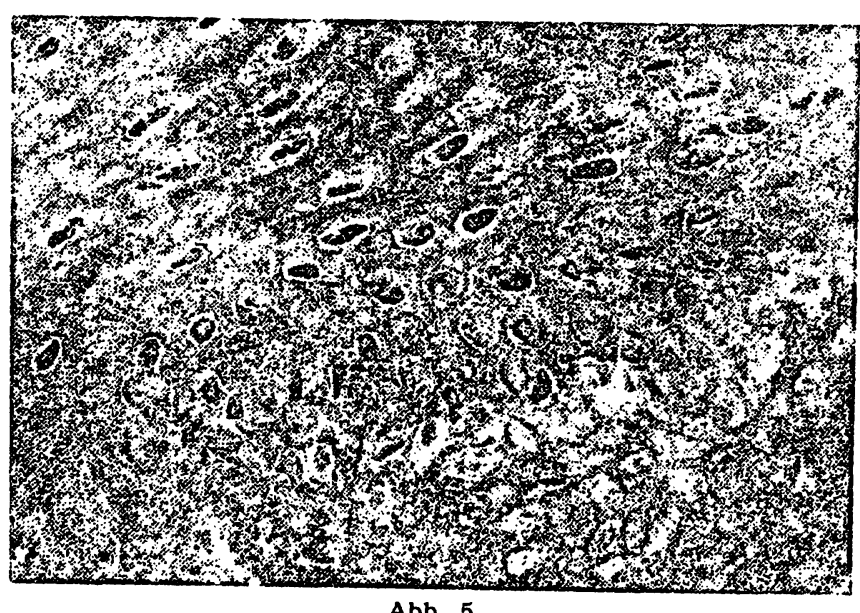

Abb. 5

Spezifische Fluoreszenz am Zellkernbereich epidermaler Zellen; Patientenserum Nr. 1. Objektiv 40 fach. Zur Methodik siehe Legende
Abbildung 2. 
garis typischerweise gefunden wird, zeigt die Abbildung 4.

Bei einem Vergleich zwischen menschlicher Epidermis und Meerschweinchenlippe erwies sich die letztgenannte ebenso geeignet als „antigenes Substrat" zum Nachweis von Antikörpern gegen Strukturen in der Epidermis, was BEUTNER und Mitarbeiter (9) bereits 1967 zeigen konnten. In einigen Seren, auch darauf wiesen diese Autoren hin, finden sich daneben offensichtlich noch weitere Antikörper, die gegen Strukturen im und am Zellkernbereich gerichtet sind (Abb. 5). Diese konnten bei einem Patienten (Nr. 1) sowohl im Ausgangsserum als auch in $\operatorname{der} \gamma$ - und in $\operatorname{der} \gamma_{3}^{\prime}$-Fraktion nachgewiesen werden.

Die Nachweisbarkeit dieser Antikörper variiert offensichtlich in verschiedenen Spezies (Menschen-, Meer- schweinchenhaut) und auch interindividuell beim Menschen (Haut von verschiedenen Patienten mit verschiedenen Erkrankungen). In der Regel sind sie nur in unverdünnten bis schwach verdünnten Seren nachweisbar. In einer Verdünnung, in welcher Antikörper gegen Strukturen im und am Zellkernbereich nicht mehr sichtbar gemacht werden können, läßt sich der Basalmembran-Antikörper deutlicher darstellen. Daraus ergibt sich ein Hinweis dafür, daß diese zusätzlich beobachteten Antikörper sich möglicherweise hemmend auf den Nachweis des Basalmembran-Antikörpers auswirken können. Vielleicht handelt es sich nicht um differente Eiweiße, sondern um einen einheitlichen Antikörper, der in Abhängigkeit (Spezies und Individuen) in unterschiedlichem Maße gebunden wird.

\section{Literatur}

1. Beutner, E. H. und R. E. Jordan, Proc. Soc. exp. Biol. Med. (N. Y.) 117,505 (1964). - 2. Beutner, E. H., W. F. Lever, E. Witebsky, R. Jordan und B. Chertock, J. Amer. Med. Ass. 192, 682 (1965). - 3. SAMs, W. M., Arch. Derm. 102, 485 (1970). 4. Kickhöfen, B., D. K. Hammer und D. Scheel, Hoppe Seyler's Z. physiol. Chem. 349, 1755 (1968). - 5. КонN, A., Nature (London) 183, 1055 (1959). - 6. Weichselbaum, T. E., Amer. J. clin. Path. (Techn. Sect.) 10, 40 (1946). - 7. Warburg, O. und W. Christian, Biochem. Z. 310,384 (1942). - 8. Weller, T. H. und A. H. Coons, Proc. Soc. Exp. Biol. Med. (N. Y.) 86, 789 (1954). - 9. BeutNer, E. H., E. L. Rhodes und E. J. Holborow,
Clin. exp. Immunol. 2, 141 (1967). - 10. Grabar, P. und P. Burtin, Immunelektrophoretische Analyse. Elsevier Publishing Company, Amsterdam-London-New York (1964). - 11. MaNcini, G., J. P. Vaerman, A. O. Carbonara und J. F. Heremans, A single radial diffusion method for the immunological quantitation of proteins. In PeEters, H., Proc. XI. Coll. Protides of the Biological Fluids, S. 370. Elsevier Publishing Company, Amsterdam-London-New York (1964). - 12. GroB, P. J. und T. M. InDERBITZn, J. Invest. Derm. (Baltimore) 49, 282 (1967). 13. Schwartz, R. S., zit. nach l. c. (12). - 14. FAHEX, J. L. und E. M. McKeLveY, J. Immunol. 94, 84 (1965).

Priv.-Doz. Dr. H. Bockendahl Dr. W. REMY 1000 Berlin 65 Augustenburger Platz 1 